\title{
Structural Health Monitoring (SHM) of Space Structures
}

\author{
Aswin Haridas ${ }^{1, a,{ }^{*}}$ and Holger Speckmann ${ }^{1, b}$ \\ 1 Testia GmbH, Cornelius-Edzard-Str. 15 - 28199 Bremen, Germany \\ aaswin.haridas@airbus.com, bholger.speckmann@airbus.com
}

\begin{abstract}
Keywords: Structural Health Monitoring, Space Structures, Asset Monitoring, Optical Fiber Sensors, Future Technologies
\end{abstract}

\begin{abstract}
Recent years have seen an increased interest in exploring outer space for space tourism or for unmanned or manned planetary explorations. The captivated interests among various stakeholders to employ advanced technologies to meet the requirements of these missions have necessitated the use of newly developed asset monitoring systems to ensure robustness and mission reliability. Although, Non-Destructive Testing (NDT) methods provide sufficient information about the state of the structure at the time of inspection, the need for continuously monitoring the health of the structure throughout the mission has asserted the use of Structure Health Monitoring (SHM) technologies to increase the levels of safety and thereby, reducing the overall mission costs. However, since the implementation of SHM technologies for space missions can be affected by several factors including, environmental conditions, measurement reliability and unavailability of adequate standards, additional considerations on its employability must be reconsidered. This article demonstrates a structured approach to compare the capabilities of some of the most promising SHM technologies in consideration of these influential factors. Additionally, remarks on the feasibility of employing these SHM technologies and the role they could play in such critical missions would be elaborated.
\end{abstract}

\section{Introduction}

Over the past few decades, several stakeholders in the space exploration sector have thoroughly understood and implemented strategies for monitoring mission critical structures to ensure improved levels of mission safety, reliability \& affordability [1,2]. Prominent stakeholders including ESA and NASA have therefore incorporated Structural Health Monitoring (SHM) sensors and systems for on-board condition monitoring, fault detection and to generate prescriptive recovery actions. In addition, in order to pave the way towards having reusable space assets, reducing the vehicle downtime, operating costs and the maintenance costs, these stakeholders are also investigating approaches to understand and predict the lifetime of a mission critical structure.

SHM was born from the conjunction of several techniques that share a common basis with Non-Destructive Testing (NDT). In fact, by permanently installing and integrating some of the NDT sensor technologies onto the structure of interest, they could be converted into SHM techniques. Some of the most commonly used SHM technologies include, conventional strain gauges, fiber optic sensors and acoustic sensing techniques, among many others [3]. Since the fundamental basis of each of these technologies are different, their performance is highly dependent on the use-case under consideration. For monitoring the health of space structures, one such performance hindering parameter is the ambient space condition itself (ambient temperatures vacuum, cosmic radiation and electromagnetic emissions) [4]. Therefore, when selecting appropriate SHM techniques for such application, the capability of the sensors must be fully 
understood and their performance must be optimized to compensate for the influences of the space environment.

Even though, several studies investigate the performance of SHM technologies for monitoring the assets in a space environment, the majority of the research have looked into the performance of an individual SHM technology [1, 2, 5, 6]. Furthermore, since the application of SHM for space assets is a relatively new innovation, additional developments, especially for standardizing the behavior of such sensors and systems are quintessential. This would require the creation of standards to define the minimum requirements for a SHM system, which is nonexistent.

In this context, this paper investigates into the most commonly used SHM technologies and compares their performance based on a set of requirements. These requirements have been derived from several factors including, environmental conditions, measurement reliability and the technology maturity. In addition, since the system requirements for space applications can be comparable with (to an extent) the ones defined for fixed wing aircrafts, we have used the SAE ARP 6461 standards for our study [7]. This standard provides, "Guidelines for implementation of structural health monitoring on fixed wing aircrafts", which could be relevant for applications including (not limited by), helicopters, space crafts and launchers. Since it is impossible to compare the performance of all of the available SHM systems for space asset monitoring, we have limited our scope to identify and compare some of the most commonly used and promising sensor technologies. The work done in this article is a follow-up to our previous work, wherein we had evaluated the performance of fiber optic sensors, Piezoelectric Wafer Active Sensors (PWAS), Acoustic Emission (AE) sensors and conventional strain gauges in meeting the requirements posed by the space sector [8]. Upon studying the capabilities of each of these sensor technologies and its alignment with the requirement list for monitoring operation parameters \& damage parameters, fiber optic sensors were determined to be the most promising. Although, PWAS (e.g. SMART sensors from Acellent ${ }^{\mathrm{TM}}$ ) were observed to be not so capable in monitoring the operational parameters, their ability in monitoring damage parameters, especially in the case of composite structures, provide a promising solution for the future of monitoring futuristic space structures. Therefore, the analysis and evaluation presented in this paper compares the capability of the fiber optic sensors with two additional promising sensor technologies for monitoring the health of aerospace structures, namely, Comparative Vacuum Monitoring (CVM) and Surface Acoustic Waves (SAW). The next few sections would detail the capabilities of each of these sensor technologies which would be followed by the definition of the set of requirements. The aim of the analysis is to determine one (or more) promising technology (or technologies), which can be envisaged to revolutionize the space industry in the near future by setting very high standards for safety, reliability and robustness.

\section{Optical Fiber Sensors}

The use of sensors based on fiber optic technology for strain sensing, vibration monitoring, temperature measurements etc., have gained momentum due to its higher sensitivity \& form factor. In essence, a fiber optic sensor fundamentally consists of an optical source that is optically aligned with a single mode fiber optic cable. The relationship between the optical properties of the signal (light) transmitted through the optical fiber and the measurement parameter is used to measure the structural condition \& performance in real-time. Depending on the sensing mechanism, fiber optic sensors can be classified into three broad categories, namely, single point sensors, multiplexed sensors and distributed sensors $[9,10]$.

Single point sensing technology consists of one small, durable and highly accurate measurement device connected to a high-bandwidth fiber optic cable. Fiber Bragg Grating (FBG), 
which is one of the most commonly used fiber optic sensing system, is an example of this category. In essence, a FBG sensing unit is manufactured by modifying a single-mode optical fiber using a UV laser. The resultant germanium-doped microstructures creates a periodic variation in the refractive index altering the optical properties of the coherent light source passing through the optical fiber. The periodic variations in refractive index, also called Bragg gratings inherently reflect a very narrow wavelength, while transmitting all the other wavelengths through the optical fiber. The wavelength band reflected by the Bragg gratings are strongly dependent on the grating period and thus any external perturbations that affect the same can be correlated with the shift in the reflected wavelength band. Using an instrument, which is known as an interrogator (i.e. the data acquisition device), the shift in the wavelength is recorded. From an application standpoint, these sensors are multiplexed and located at strategic locations along the fiber to create a series of connected units for a quasi-distributed measurement [9].

A distributed fiber optic sensor on the other hand relates the changes in scattered light along the entire length of the optical fiber. The change in scattered light is used to determine the local variation of physical quantities (strains or temperature) [10]. In this case, the entire fiber acts as the sensor element. Depending on whether the mechanism of scattering the light within an optical fiber is elastic or inelastic, the sensing technique can be classified, Rayleigh, Brillouin and Raman scattering techniques. Whilst Rayleigh scattering is a physical phenomenon caused due to non-propagating density fluctuations (scattered power is proportional to the input power), Brillouin and Raman scattering result from inelastic physical phenomenon causing large degrees of frequency shifts. Considering the advantages of each of the techniques in the measurement of physical quantities, Rayleigh and Brillouin scattering are being widely investigated for strain measurement applications and Raman scattering is being studied for temperature measurements. Fiber optic sensing technologies have seen widespread applications for monitoring the operational parameters (strains \& temperatures) in several industries, including, infrastructure, aeronautical, automotive and for mainstream industrial processes (not limited to) $[9,10]$.

To ensure the accuracy, repeatability and stability of the measurements made by the fiber optic sensor, multiple parameters play an important role. In the case of applications that require the sensor to be mounted on the surface of the structure of interest, the choice of adhesive is of prime importance. This coating must be able to protect the sensor from mechanical and environmental influences, maximizing its performance. Generally, the sensors are coated using a polymer, acrylate or polyamide base. Parameters including, the choice of adhesive for mounting the fiber, the implementation strategy (externally mounted or embedded) and sensor orientation (important for composite structures) must be optimized $[4,11]$.

These sensors, which are also minimally influenced by EMI induced noise, are comparatively inexpensive and lightweight. Combined with the possibility of performing measurements over long distances, the applicability of the same for a space environment is promising. However, the lack of suitable standards and the need for discriminating the wavelength shift due to strains and temperature necessitate trials and field tests prior to implementing the same for space applications in the near future [9-11].

\section{Surface Acoustic Wave (SAW)}

Typically, a SAW sensor constitutes a piezoelectric substrate on which a periodic comb-shaped inter digital transducer (IDT) pattern is developed using a photolithographic process. The electrodes are generally manufactured using inert alloys, for example, $\mathrm{Au}, \mathrm{Cr} / \mathrm{Au} / \mathrm{Cr}$, etc. When an AC voltage is applied across the IDTs, an acoustic wave is generated which travels across the surface of the crystal (perpendicular to the IDT). The generated acoustic waves are confined to the 
surface of the substrates and they have a penetration depth of a few wavelengths. A basic SAW sensor that contains two IDTs on a piezoelectric surface is shown in Figure 1. The input IDT generates the acoustic wave which is collected/reflected by the output IDT [12].

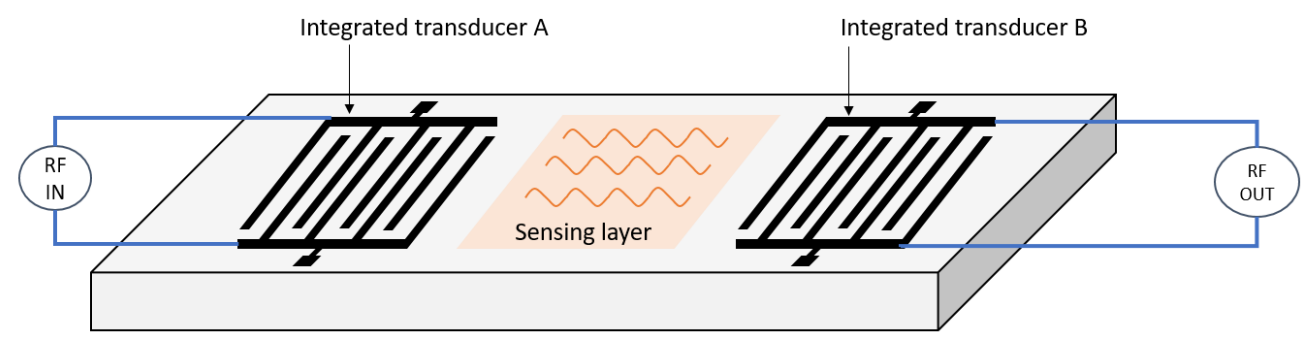

Fig. 1. A basic SAW sensor (Adapted from [12])

Based on the sensing mechanism, a SAW sensor can be classified into two broad categories, namely, direct or indirect. While the former directly measures the variation of the physical quantities such as temperature, pressure, torque, etc., the second type the SAW device transforms the output of another direct sensor to the electrical signal convenient for further processing. Both types can be accessed wirelessly. Furthermore, the operational modality of the SAW sensors have been demonstrated both in an active and passive mode. In case of the former, the SAW sensors would require batteries to be placed preferably in close proximity. However, for applications which require the sensor to operate in a high temperature environment $\left(\sim 1500^{\circ} \mathrm{C}\right)$, for example, within a propulsion system or inside gas pipes, the passive (wireless without batteries) modality would be preferred. In case of the other temperature extreme, i.e. for cryogenic environments, the successful operation of the SAW sensors have been studied and demonstrated [13]

Since these sensors can withstand high temperatures up to about $1500{ }^{\circ} \mathrm{C}$ and have a high resistance to ionizing radiation (demonstrated tolerance- upto $10 \mathrm{Mrad}$ ) without power loss, they are a promising contender for space applications [13]. Although SAW sensors provide several benefits for such critical applications, one of the main challenges for the continuous wireless operation of these sensors is power generation. Often, batteries are not recommended to be used due to the inaccessibility of the inspection area or due to the exposure to large temperature extremes. Which is why, these sensors are mostly implemented in the passive mode. Additional factors that affect the measurements made by the SAW sensors include, a) dependence on vibration and mechanical shocks, b) dependence on the temperature variation (causing the output frequencies to shift; can be compensated) and c) the bandwidth of the Radio Frequency (RF) communication channel (limiting the number of sensors that can be wirelessly connected). In addition to these factors, the importance of defining a certification regime for wireless sensor networks is an additional challenge (considering the interference of wireless devices with other electronics) [12-13].

\section{Comparative Vacuum Monitoring (CVM)}

A CVM sensor is a simple pneumatic sensor technology that has been used to continuously and autonomously monitor the onset of cracks. These sensors are permanently adhered onto the structure of interest to monitor their critical regions. The basic principle of a CVM sensor is to maintain a small volume in low vacuum that is extremely sensitive to ingress of air and thus highly sensitive to leakage. The working principle of a typical CVM sensor is shown in Figures 2 (a) and 2 (b). In both these figures, the actual sensing part, which is a sensor pad can be visible onto which several fine galleries containing a low vacuum are incorporated, alternative with galleries at the 
nominal atmospheric pressure. To maintain the vacuum in the respective galleries, a vacuum source is used, which can be seen in Figures 2 (a) and 2 (b). In addition, to measure the differential pressure between these galleries, a differential pressure flow meter is incorporated. In the absence of a crack, the differential flow pressure values are show the baseline values (see Figure 2 (a)). However, if a crack develops within the structure (see Figure 2 (b)), the vacuum galleries are disturbed by the ingress of air from the galleries at nominal atmospheric pressure. This in turn would be measured as a drop in pressure differential by the flow meter. Subsequently, using the data from the flow meter, control systems and software management tools are engaged to process the data. Further, the final information about the position and size of the crack is visually presented to the user. Typically, with gallery spacing of $250 \mu \mathrm{m}$ a crack as small as $100 \mu \mathrm{m}$ can be identified [14-16].

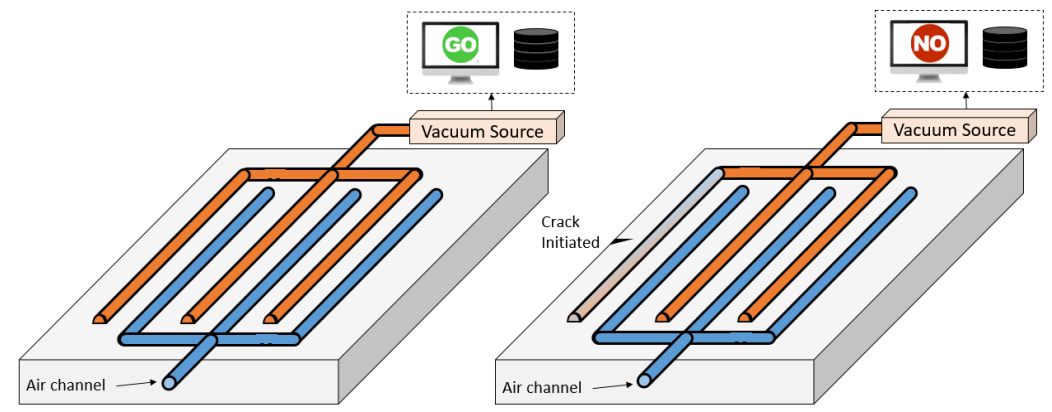

Fig. 2. The working principle of CVM sensors, (a) normal state, (b) during the onset of a crack (adapted from [14]).

Being a relatively simple technique that require no electrical excitation, the CVM sensors have been widely employed for the inspection of aircraft structures and to address the critical infrastructure surety needs [14]. This said, the application range of these sensors are not limited and they span several industries, including, mining structures, trains \& subway vehicles, cars, trucks and heavy machinery, to name a few [15-16]. Some of the benefits offered by CVM include, a) its high maturity levels for immediate implementation (successfully tested and validated in actual test environments), b) the possibility of incorporating self-diagnostics, c) the possibility of improving the system portability for in-service inspections and d) its use-case flexibility, which allows these sensors to be also used for applications that necessitates embedded sensing (for e.g. monitoring of aircraft riveted lap and butt joints or those that require a through the thickness assessment). However, since the CVM sensor detects the initiation of defects based on measurement deviation from a base-line reading from the pressure flow meter, the same must be thoroughly calibrated before the test to avoid any false positives. Therefore, if there is a known defect on the location of sensor application, the detector would measure a non-zero base value. This remains one of the disadvantages of the sensor apart from the installation effort. Also, it must be noted that the sensitivity of the sensor is dependent on the gallery wall thickness [15].

\section{Feasibility Analysis Using a Decision Matrix}

To compare the capabilities of the SHM technologies, a decision matrix stating the list of requirements were compiled based on prior experience along with the inputs from the ArianeGroup. This decision matrix is similar to the one prepared in our previous work [8]. In general, the aim is to assess the capabilities of the SHM technologies based on the requirements in system parameters, condition monitoring parameters, damage monitoring parameters and environmental parameters. In order to achieve a fair comparison between the techniques, a weighted decision matrix would be prepared. In order to do so, a ranking score would be assigned 
and each of the technologies would be ranked depending on its performance in comparison with the desired state.

Table 1. Decision matrix definition

\begin{tabular}{|c|c|c|c|c|}
\hline \multirow{2}{*}{ Parameters } & \multirow{2}{*}{$\begin{array}{l}\text { Desired } \\
\text { State }\end{array}$} & \multicolumn{3}{|c|}{ Decision Matrix Score } \\
\hline & & 1 & 0.5 & 0 \\
\hline \multicolumn{5}{|l|}{ System Parameters } \\
\hline Total weight & Low & Low & Medium & High \\
\hline Installation effort (cost) & Low & Low & Medium & High \\
\hline Power consumption & Low & Low & Medium & High \\
\hline Transmitting data to a remote location & Yes & Yes & & No \\
\hline Self-diagnostic ability & Yes & Yes & & No \\
\hline Possibility of repair for accessible areas & Yes & Yes & Partially** & No \\
\hline $\begin{array}{c}\text { Measurement accuracy, repeatability, resolution \& } \\
\text { robustness }\end{array}$ & High & High & Medium & Low \\
\hline Monitoring area & Large & Large & & Small \\
\hline Sensor cost/ unit area & Low & Low & Medium & High \\
\hline Data acquisition time & Low & Low & Medium & High \\
\hline Sensor application location* & $\begin{array}{l}\text { Ext. \& } \\
\text { emb }\end{array}$ & $\begin{array}{l}\text { Ext. \& } \\
\text { emb. }\end{array}$ & $\begin{array}{l}\text { Ext. \& } \\
\text { semi emb. }\end{array}$ & Only ext. \\
\hline TRL level & High & $\begin{array}{l}\text { TRL } \\
8-9\end{array}$ & TRL 6-7 & $\mathrm{TRL}<6$ \\
\hline \multicolumn{5}{|l|}{ Condition Monitoring Parameters } \\
\hline Strain measurement range & High & Large & & Small \\
\hline Temperature measurement range & High & Large & & Small \\
\hline Distributed sensing capability & Yes & Yes & & No \\
\hline \multicolumn{5}{|l|}{ Damage Monitoring Parameters } \\
\hline Defect measurement sensitivity & High & Yes & $\begin{array}{l}\text { Yes w/o } \\
\text { location }\end{array}$ & No \\
\hline Identifying the defect type & Yes & Yes & & No \\
\hline Environmental Parameters & & & & No \\
\hline Resistance to cosmic radiation with high energy & High & Yes & & No \\
\hline Resistance to electromagnetic radiation & High & Yes & & No \\
\hline Usable in vacuum $\left(10^{-10}\right.$ and $\left.10^{-11} \mathrm{Atm}\right)$ & Yes & Yes & & No \\
\hline Usable in low gravity & Yes & Yes & & High \\
\hline
\end{tabular}

* Ext. and emb. - External and embedded **Depends on whether the sensor is embedded and the use case

To determine the capability of the SHM techniques, a ranking score (based on how the technique satisfies a requirements) is assigned, which is combined with a weighting factor. The results of this analysis is shown in Table 2.

From an initial analysis of the weighted sum, the capabilities offered by the fiber optic sensors dominate the other two SHM technologies. Apart from being immune (partially; requires further tests) to ambient conditions, the main advantage of fiber optics sensors arise due to its multiplexing capability. Having several sensor points distributed over the length of an optical fiber, one can minimize the overall weight per sensor (relative to other sensors). Furthermore, the overall costs for procuring and implementing fiber optic sensors are also minimal compared with the CVM and SAW sensors. In comparison, the capabilities of SAW sensors are observed to be comparable to the fiber optic sensors. However, essentially being a point sensor, the advantages 
they offer are diluted (increased weight $\&$ installation effort and cost) in comparison to the fiber optic sensors when considering larger scales of implementation. CVM sensors on the other had offer minimal advantages in meeting the requirements posed by the space sector. Although CVM sensors are easy to use and offer self-diagnostic capabilities, they are seen to minimally add value due to the implementation effort (higher installation cost) and due to their non-immunity for applications in the space environment (requires further tests to confirm).

Table 2. Evaluation table

\begin{tabular}{|c|c|c|c|c|}
\hline $\begin{array}{l}\text { a) Fiber op } \\
\text { b) CVM }\end{array}$ & sensors & & $\mathrm{c}$ & SAW \\
\hline Parameters & $\begin{array}{l}\text { Weighing } \\
\text { factor }\end{array}$ & a) & b) & c) \\
\hline Total weight & 5 & 1 & 0 & 0 \\
\hline Installation effort (cost) & 3 & 0.5 & 0 & 0.5 \\
\hline Power consumption & 3 & 0.5 & 0.5 & 0.5 \\
\hline Transmitting data to a remote location & 4 & 1 & 1 & 1 \\
\hline Self-diagnostic ability & 3 & 0 & 1 & 0 \\
\hline & 4 & 0.5 & 1 & 1 \\
\hline $\begin{array}{c}\text { Possibility of repair for accessible areas** } \\
\text { Measurement accuracy, repeatability, resolution } \\
\& \text { robustness } *\end{array}$ & 5 & 1 & 1 & 1 \\
\hline Monitoring area & 2 & 0 & 0 & 0 \\
\hline Sensor cost/ unit area & 2 & 1 & 0 & 0 \\
\hline Data acquisition time & 2 & 1 & 0 & 1 \\
\hline Sensor application location* & 3 & 1 & 0.5 & 0 \\
\hline TRL level & 4 & 0.5 & 1 & 0 \\
\hline Strain measurement range & 5 & 1 & 0 & 1 \\
\hline Temperature measurement range & 5 & 1 & 0 & 1 \\
\hline Distributed sensing capability & 3 & 1 & 0 & 0 \\
\hline Defect measurement sensitivity & 5 & 0.5 & 1 & 1 \\
\hline Identifying the defect type & 5 & 0 & 0 & 1 \\
\hline Resistance to cosmic radiation with high energy & 5 & 1 & $0^{*}$ & 1 \\
\hline Resistance to electromagnetic radiation & 5 & 1 & 0 & $1 *$ \\
\hline Usable in vacuum $\left(10^{-10}\right.$ and $\left.10^{-11} \mathrm{Atm}\right)$ & 5 & $0^{*}$ & $0^{*}$ & $0 *$ \\
\hline Usable in low gravity & 5 & $0 *$ & $0^{*}$ & $0 *$ \\
\hline$\underline{\text { Total }}$ & & 53.5 & 28 & 48 \\
\hline
\end{tabular}

* Requires further tests to confirm $\quad * *$ considering the popularity of embedding the sensors

\section{Summary}

This work was inspired by the rapid growth of the space exploration sector and due to the rising criticality in monitoring the health of mission critical structures. In this article, three of the most commonly used/ promising SHM techniques are introduced and compared with each other on the basis of a set of requirements prepared from our prior experience and inputs from the ArianeGroup. Three sensors, namely, fiber optic sensors, CVM sensors and SAW sensors are compared considering system parameter, operational parameters (condition and damage monitoring) and environmental parameters. Using a weighted evaluation, fiber optic sensors were observed to be the most promising due to their multiplexing capabilities. Even though SAW 
sensors are observed to be comparable to the performance of fiber optic sensors, the complexities added during the large scale implementation of the same is observed to hinder its overall performance. CVM sensors on the other hand fail to be comparable with the capability of fiber optic sensors \& the SAW sensors. Although CVM sensors offer several advantages for aeronautical applications, there is a sufficient gap in understanding its capability for space applications, which could be a potential interest domain for further research. In conclusion, the research presented in the article covers a small scope of available sensors. The intention of the same is to spark interests and provide a strategy in technology comparison that could facilitate future space missions, making them safer, reliable and dependable.

\section{References}

[1] W. L Richards, E. Madaras, W.H. Prosser, G. Studor, NASA Applications of Structural Health Monitoring Technology, 9th International Workshop on Structural Health Monitoring, September 10-12, (2013).

[2] M. Iain, N. Karafolas, Fiber optic sensing in space structures: the experience of the European Space Agency, In 17th International Conference on Optical Fibre Sensors, 5855 (2005), 262-269. International Society for Optics and Photonics. https://doi.org/10.1117/12.623988

[3] H. Speckmann, R. Henrich, Structural health monitoring (SHM)-overview on technologies under development, In Proc. of the World Conference on NDT, Montreal-Canada, (2004).

[4] B. Daniel, C.P Fritzen, A. Güemes, Structural health monitoring. Vol. 90. John Wiley \& Sons, 2010 .

[5] M. Simone, G. Tumino, P. Gaudenzi. "Structural health monitoring for future space vehicles." Journal of intelligent material systems and structures 17, no. 7 (2006): 577-585. https://doi.org/10.1177/1045389X06059077

[6] I. Tansel, P. Chen, X. Wang, A. Yenilmez, B. Ozcelik, Structural health monitoring applications for space structures, In Proceedings of 2nd International Conference on Recent Advances in Space Technologies, 288-292 (2005), IEEE.

[7] F. Peter. "New guidelines for implementation of structural health monitoring in aerospace applications." SAE International Journal of Aerospace 6, no. 2013-01-2219: 525-533 (2013). https://doi.org/10.4271/2013-01-2219

[8] A. Haridas, C.M Giraldo, H. Speckmann, Structural Health Monitoring (SHM) goes to space: submitted to 10th European Workshop on Structural Health Monitoring, EWSHM 2020. https://doi.org/10.1007/978-3-030-64594-6_39

[9] C.E. Campanella, A. Cuccovillo, C. Campanella, A. Yurt, V. Passaro, Fibre Bragg grating based strain sensors: review of technology and applications, Sensors 18, no. 9 (2018), 3115. https://doi.org/10.3390/s18093115

[10] B. Glisic, Distributed fiber optic sensing technologies and applications-an overview, ACI Spec. Publ 292 (2013): 1-18.

[11] C.M. Giraldo, J.Z. Sagredo, J.M Gómez, P. Corredera, Demonstration and methodology of structural monitoring of stringer runs out composite areas by embedded optical fiber sensors and connectors integrated during production in a composite plant. Sensors, 17(7), 1683 (2017). https://doi.org/10.3390/s17071683 
[12] M.F. Hribšek, D.V. Tošić, M.R. Radosavljević, Surface acoustic wave sensors in mechanical engineering. FME transactions, 38(1) (2010), 11-18.

[13] W.C. Wilson, D.F. Perey, G.M. Atkinson, R.O. Barclay, Passive wireless SAW sensors for IVHM. In 2008 IEEE International Frequency Control Symposium, 273-277, IEEE. https://doi.org/10.1109/FREQ.2008.4623003

[14] H. Stehmeier, H. Speckmann, Comparative vacuum monitoring (CVM). In Proceedings of the 2nd European Workshop on Structural Health Monitoring, Munich, Germany, (2004) 7-9.

[15] D. Roach, Real time crack detection using mountable comparative vacuum monitoring sensors, Smart structures and systems 5, no. 4 (2009): 317-328. https://doi.org/10.12989/sss.2009.5.4.317

[16] D. Roach, Use of Comparative Vacuum Monitoring Sensors for Automated Wireless Health Monitoring of Bridges and Infrastructure, United States: N. p., 2018. Web.

https://doi.org/10.1201/9781315189390-372 